\title{
Equações para estimar área foliar de folíolos de Acrocomia aculeta
}

\author{
Clenilso Sehnen Mota ${ }^{1}$, Helio Garcia Leite ${ }^{2}$, Marco Antonio Oliva Cano ${ }^{3}$ \\ ${ }^{1}$ Instituto Federal Goiano, Rodovia Sul Goiana, Km 1, CEP 75901-970, Rio Verde, GO, Brasil \\ ${ }^{2}$ Universidade Federal de Viçosa, Departamento de Engenharia Florestal, Av. P.H. Rolfs, s/nº, CEP 36570-000, Viçosa, MG, Brasil \\ ${ }^{3}$ Universidade de Vila Velha, Rua Comissário José Dantas de Melo, n² 21, Boa Vista, CEP 29102-770, Vila Velha, ES, Brasil
}

\begin{abstract}
"Autor correspondente:
csm.sehnen@gmail.com
\end{abstract}

Termos para indexação:

Método não destrutivo

Ajuste de equações

Superfície foliolar

Index terms:

Non-destructive method

Equations adjustment

Leaflet surface

Histórico do artigo:

Recebido em 01/04/2014

Aprovado em 27/08/2014

Publicado em 10/10/2014

doi: 10.4336/2014.pfb.34.79.684
Resumo - O objetivo desse estudo foi desenvolver um modelo estatístico empírico, para estimar a superfície foliar, por um método não destrutivo, de folíolos de plantas jovens e adultas da palmeira macaúba. Foram coletados oito folíolos por folha, quatro em cada lado e duas folhas por plantas, em lados opostos, totalizando 16 folíolos em cada uma das cinco plantas, por estádio fenológico (jovens e adultas). Foram avaliados um total de 80 folíolos para cada estádio fenológico. Individualmente, cada folíolo teve sua área (AF), comprimento (C) e largura (L) mensurados. Foram testados modelos lineares, com e sem o intercepto, e um modelo potencial, empregando as variáveis independentes $\mathrm{C}, \mathrm{L}$ e $\mathrm{C}^{*} \mathrm{~L}$. As equações com coeficiente de determinação inferior a 0,90 foram descartadas e o uso da variável combinada $C *$ L resultou em maior exatidão e melhor distribuição dos resíduos, ao empregar a relação funcional $y=\beta 0 x^{\beta 1}$. Assim, $a$ área de folíolos de macaúba pode ser estimada pela equação $\mathrm{AF}=0,4683 \mathrm{CL}^{1,1104}$.

\section{Equations to estimate leaf area of Acrocomia aculeta leaflets}

\begin{abstract}
The aim of this study was to develop an empirical statistical model to estimate leaf surface by a non-destructive method of leaflets in young and mature plants palm macaúba. Eight leaflets per leaf, four on each side and two leaves per plant on opposite sides, total 16 leaves were collected from each of five plants per growth stage (juvenile and adult) were collected. Thus obtaining a total of 80 leaflets for each phenological stage. Individually each leaflet had its area (LA), length (L) and width (W) measured. Linear models with and without the intercept, and a potential model were tested using the independent variables W, L and combined (WL). Equations with coefficient of determination less than 0.90 , were discarded and the combined use of the WL variable resulted in greater accuracy and better distribution of residuals, by employing the functional relationship $y=\beta 0 x^{\beta 1}$. Thus, the area of leaflets macaúba can be estimated by $\mathrm{LA}=0.4683 \mathrm{WL}^{1,1104}$ equation.
\end{abstract}




\section{Introdução}

A palmeira macaúba [Acrocomia aculeata (Jacq.) Lodd. ex Mart.] apresenta ampla utilidade e tem despertado interesse socioeconômico por sua capacidade de produção de óleo vegetal. Também são relatados usos alimentícios, importância ambiental e na indústria de produtos manufaturados, como fármacos, cosméticos, resinas, lubrificantes, entre outros. Recentemente, com o advento do uso de óleos vegetais para produção de biodiesel, a macaúba tem recebido especial atenção por sua capacidade produtiva de óleo, comparável somente ao dendê (Elaeis Guineenses Jacq.). Além disso, é uma planta adaptada e nativa de ambiente árido, como o Cerrado (Mota et al., 2011).

Em estudos de ecofisiologia vegetal, a estimativa da área foliar auxilia na verificação da superfície fotossintética, o que permite obter indicadores importantes para o entendimento das respostas das plantas a fatores ambientais ou de qualquer outro fator que influencie estas respostas (Lopes et al., 2004). Sendo assim, a área foliar é uma indicadora da taxa transpiratória, taxa de assimilação de $\mathrm{CO}_{2}$, taxa de liberação de $\mathrm{O}_{2}$ e vigor das plantas. A partir do índice de área foliar também é possível estimar a produtividade. Por estas razões, é fundamental estabelecer metodologias que visem avaliar os parâmetros necessários para estimar a área foliar, particularmente em condições de campo (Welles, 1990).

A estimativa da área foliar pode ser obtida pelo uso de dois métodos, o destrutivo e o não destrutivo. Os métodos destrutivos são simples e precisos, todavia apresentam o inconveniente de demandar muito tempo (Lopes et al., 2004), além de provocar a destruição da área foliar, o que impossibilita o seu acompanhamento durante o ciclo da cultura. A metodologia mais simples, proporcionada pelos métodos não destrutivos, é baseada na mensuração de elementos da lâmina foliar e sua comparação com a área foliar. Assim, a área foliar pode ser estimada por meio de equações de regressão, entre a área foliar real e os parâmetros dimensionais lineares das folhas. $\mathrm{O}$ método não destrutivo permite acompanhar o crescimento, expansão e incremento da área foliar da mesma planta até o final do ciclo ou do ensaio, além de ser rápido e preciso. Esse método tem especial importância no caso da macaúba, onde a taxa de renovação da área foliar é lenta, principalmente durante a fase juvenil. Os modelos estatísticos são variados e são utilizados para estimar indiretamente a área foliar de diversas plantas tropicais.
A área de uma folha ou folíolo individualmente pode ser estimada a partir da soma do comprimento das nervuras principais (Campostrini \& Yamanishi, 2001; Gonçalves et al., 2002; Malagi et al., 2010), do comprimento de lâminas foliares e/ou largura máxima (Bianco et al, 2005; Antunes et al., 2008), ou com base na massa das folhas (Sepúlveda \& Kliewer, 1983). O método usado depende da espécie, cultivar e das características de folha, tais como forma, número de lobos, forma da lâmina foliar, etc. É importante que a amostra seja representativa, para que obtenha alta precisão.

Esse trabalho teve como objetivo o desenvolvimento de um modelo estatístico empírico de estimativa da superfície foliar por um metodo não destrutivo, de folíolos de plantas jovens e adultas da palmeira macaúba.

\section{Material e métodos}

Os folíolos foram coletados em plantas jovens e adultas. Os folíolos das plantas jovens foram coletados em plantas cultivadas em casa de vegetação na Universidade Federal de Viçosa (2045'S, 42 $\left.52^{\circ} \mathrm{W}\right)$, em vasos com capacidade de 150 L. Essa plantas apresentavam 18 meses de idade e cerca de cinco folhas definitivas e sadias, que recebiam irrigações diárias. Os folíolos das plantas adultas foram coletados no Município de Piranga, MG $\left(20^{\circ} 41^{\prime} \mathrm{S}, 43^{\circ} 18^{\prime} \mathrm{W}\right)$, em plantas sadias com frutos, e de ocorrência natural em pastagem, com cerca de $10 \mathrm{~m}$ de altura.

Foram coletados oito folíolos por folha, sendo quatro em cada lado da folha, e em duas folhas por plantas, uma em cada lado da planta. No total, foram coletados 16 folíolos por planta e em cinco plantas em cada estádio fenológico (jovens e adultas). Obtevese, então, o total de 80 folíolos para cada estádio fenológico. Individualmente, cada folíolo teve sua área, comprimento (C) e largura (L) mensurados. A área foi obtida através de um integrador de área, modelo LI-3100 (LI-COR, Biosciences, Nebraska, USA). O comprimento e a largura foram obtidos com auxílio de uma régua metálica milimétrica, em que as medidas foram tomadas ao meio do folíolo.

Foram testados três modelos, sendo dois lineares (1 e 2) e um não linear (3):

$$
\begin{aligned}
& Y=\beta_{01}+\beta_{11} X+\varepsilon(1) \\
& Y=\beta_{12} X+\varepsilon(2) \\
& Y=\beta_{023} X^{\beta 13}+\varepsilon(3)
\end{aligned}
$$

Onde: $Y$ é a área foliar estimada do folíolo e $X$ é a dimensão da folha (C, L ou CL). 
As estimativas dos coeficientes dos modelos 1 e 2 foram obtidas pelo método usual de mínimos quadrados ordinários. O modelo 3 foi ajustado na forma não linear, pelo algoritmo Gauss Newton, empregando o software Statistica 10 (Statsoft, 2012). Para os modelos 1 e 2, o coeficiente de determinação $\left(\mathrm{R}^{2}\right)$ foi obtido por:

$$
R^{2}=\sum_{i=1}^{n}\left(Y_{i}-\hat{Y}_{i}\right)^{2} \sum_{i=1}^{n}\left(Y_{i}-\bar{Y}\right)^{-2}
$$

Para o modelo 3 , obteve-se uma estimativa do $R^{2}$ obtida a partir da correlação $R_{\hat{Y} Y}$, dada por:

$$
\begin{aligned}
& R_{\hat{Y} Y}=\frac{n^{-1}\left(\sum_{i=1}^{n}\left(\hat{Y}_{i}-\hat{Y}_{m}\right)\left(Y_{i}-\bar{Y}\right)\right)}{\sqrt{\left(n^{-1} \sum_{i=1}^{n}\left(\hat{Y}_{i}-\hat{Y}_{m}\right)^{2}\right)\left(n^{-1} \sum_{i=1}^{n}\left(Y_{i}-\bar{Y}\right)^{2}\right)}} \\
& \hat{Y}_{m}=n^{-1} \sum_{i=1}^{n} \hat{Y}_{i}
\end{aligned}
$$

Em que: $\hat{Y}_{m}$ é a média das estimativas de $Y ; \bar{Y}$ é a média aritmética de $Y$; e $n$ é o número de observações. $\mathrm{O}$ erro-padrão residual $\left(s_{y x}=\sqrt{Q M R e s}\right)$ mede a variação das observações quanto à curva da regressão e o QM Res é a estimativa da variância residual.

\section{Resultados e discussão}

Em plantas jovens, a área foliar dos folíolos (AFF) observada variou de $10 \mathrm{~cm}^{2}$ a $52,3 \mathrm{~cm}^{2}$, com média de $28,42 \mathrm{~cm}^{2}$. Nos folíolos provenientes das plantas adultas, a variação foi de $46,7 \mathrm{~cm}^{2}$ a $197 \mathrm{~cm}^{2}$, com média de $133,19 \mathrm{~cm}^{2}$. A menor largura dos folíolos foi de $0,7 \mathrm{~cm}$ para ambas as idades fenológicas e a máxima de $2,4 \mathrm{~cm}$ e $2,8 \mathrm{~cm}$, para jovens e adultas, e médias de $1,62 \mathrm{~cm}$ e $1,93 \mathrm{~cm}$, respectivamente. O comprimento variou de $14,8 \mathrm{~cm}$ a $35,6 \mathrm{~cm}$ e de $65,3 \mathrm{~cm}$ a $92,8 \mathrm{~cm}$, para folíolos das plantas jovens e adultas, e médias de $25,95 \mathrm{~cm}$ e $82,46 \mathrm{~cm}$, respectivamente.

A principal variável a contribuir para o incremento da AFF é o comprimento do folíolo ao longo da nervura principal, visto que a largura pouco varia, tendo um incremento de $19 \%$ na média, enquanto no comprimento esse foi de $226 \%$. A soma do comprimento das nervuras secundárias é a característica dimensional que apresenta melhor correlação com a área foliar em videiras (Gonçalves et al., 2002; Malagi et al., 2010), da nervura principal em Carica papaya (Campostrini \& Yamanishi, 2001), enquanto a largura dos folíolos tem melhor relação em meloeiro (Nascimento et al.,
2002). Com relação, ao produto entre o comprimento e largura das folhas, observa-se uma boa correlação para as seguintes espécies: Coffea sp. (Antunes et al., 2008), Cissampelos glaberrima (Bianco et al., 2002), Brachiaria plantaginea (Bianco et al., 2005), Vigna unguiculata (Lima et al., 2008) e Malus domestica (Bosco et al., 2012), sendo esse também observado no presente estudo (Tabela 1 e Figura 1).

Todos os modelos ajustados para comprimento (C), largura (L) e o produto entre comprimento e largura $\left(\mathrm{C}^{*} \mathrm{~L}\right)$ do limbo do folíolo, apresentaram alta significância, com $\mathrm{p}<0,0001$. Todavia, os modelos que utilizavam como variável independente $\mathrm{C}$ e $\mathrm{L}$ foram descartados, devido ao baixo coeficiente de correlação $\left(\mathrm{R}^{2}\right)$, ou seja, inferior a 0,90 , bem como alta soma do quadrado médio do resíduo, características essas que conferem menor precisão (Tabela 2). Nakamura et al. (2005) encontrou coeficiente de determinação superior a 0,99 na espécie Metroxylon sagu Rottb., usando a equação " $y=\beta_{0}+\beta_{1}{ }^{*} x$ ", onde y representa a AFF e $x$ a variável dimensional do folíolo ou largura máxima do mesmo, o que não corrobora com os resultados encontrados no presente trabalho que apresentou valores inferiores a 0,90. Quando a variável independente foi o produto entre o $\mathrm{C}$ e $\mathrm{L}\left(\mathrm{C}^{*} \mathrm{~L}\right)$, o valor de $\mathrm{R}^{2}$ foi igual a $0,90,0,95$ e 0,98 quando utilizou-se folíolos de plantas jovens, adultas e folíolos de plantas de ambas as idades, respectivamente. Isso independe do modelo matemático ajustado, considerando que os valores de $\mathrm{R}^{2}$ foram idênticos entre os modelos testados dentro de cada idade fenológica das plantas. Assim, as equações selecionadas explicam pelo menos $90 \%\left(\mathrm{R}^{2}>0,90\right)$ das variações observadas (Bianco et al., 2005).

$\mathrm{O}$ valor de $\mathrm{AFO} / \mathrm{AFE}$ mais próximo de um foi observado para o modelo " $\mathrm{AF}=\beta_{0}+\beta_{1}{ }^{*} \mathrm{CL}$ ", seguido pelo "AF $=\beta_{0}{ }^{*} \mathrm{CL}^{\beta 1}$ " e por último o " $\mathrm{AF}=\beta_{1}{ }^{*} \mathrm{CL}$ ", sendo que o último tende a superestimar a $A F F$, um vez que apresenta valores inferiores a um, independente da idade fenológica das plantas (Tabela 2). Lima et al. (2008), usando o modelo " $\mathrm{AF}=\beta_{0}{ }^{*} \mathrm{x}^{\beta 1}$ " para estimar área foliar de caupi, observaram que o modelo apresenta AFO/ AFE mais próximo de um quando comparado ao modelo "AF $=\beta_{0}+\beta_{1}{ }^{*} \mathrm{x}$ ", sendo $\mathrm{x}$, o comprimento, largura, soma ou produto de ambos. Demonstrou-se, assim, que para o caupi o modelo " $\mathrm{AF}=\beta_{0}{ }^{*} \mathrm{x}^{\beta 1}$ " apresenta maior correlação entre os valores estimados e os observados pelo método não destrutivo. Todavia, no presente estudo, o melhor foi o modelo " $\mathrm{AF}=\beta_{0}+\beta_{1}{ }^{*} \mathrm{CL}$ ". Infere-se esses modelos proporcionam maior precisão da estimativa. 
Tabela 1. Modelos e parâmetros estimados das equações da área foliar dos folíolos de macaúba, em função do comprimento (C), largura (L) e produto de ambos (CL), em plantas jovens (J) adultas (A) e ambas (JA).

\begin{tabular}{|c|c|c|c|c|}
\hline \multirow{2}{*}{ Modelo } & \multirow{2}{*}{ Parâmetro } & \multicolumn{3}{|c|}{ Estimativas dos parâmetros } \\
\hline & & $\mathrm{J}$ & $\mathrm{A}$ & JA \\
\hline $\mathrm{AF}=\beta_{1} * \mathrm{CL}+\varepsilon$ & $\beta_{1}$ & $0,6752 * * *$ & $0,8327 * * *$ & $0,8222 * * *$ \\
\hline \multirow{2}{*}{$\mathrm{AF}=\beta_{0}+\beta_{1}^{*} \mathrm{CL}+\varepsilon$} & $\beta_{0}$ & $-2,5007^{*}$ & $-4,2789^{\text {ns }}$ & $-8,3073 * * *$ \\
\hline & $\beta_{1}$ & $0,7293 * * *$ & $0,8576 * * *$ & $0,8793 * * *$ \\
\hline \multirow{2}{*}{$\mathrm{AF}=\beta_{0} * \mathrm{CL}^{\beta 1}+\varepsilon$} & $\beta_{0}$ & $0,4807 * * *$ & $0,7124 * * *$ & $0,4683 * * *$ \\
\hline & $\beta_{1}$ & $1,0877 * * *$ & $1,0301 * * *$ & $1,1104 * * *$ \\
\hline $\mathrm{AF}=\beta_{1} * \mathrm{C}+\varepsilon$ & $\beta_{1}$ & $1,1034 * * *$ & $1,6287 * * *$ & $1,5803 * * *$ \\
\hline \multirow{2}{*}{$\mathrm{AF}=\beta_{0}+\beta_{1} * \mathrm{C}+\varepsilon$} & $\beta_{0}$ & $-8,4749^{\mathrm{ns}}$ & $-273,0404 * * *$ & $-22,2466^{* * *}$ \\
\hline & $\beta_{1}$ & $1,4214 * * *$ & $4,9267 * * *$ & $1,9011 * * *$ \\
\hline \multirow{2}{*}{$\mathrm{AF}=\beta_{0} * \mathrm{C}^{\beta 1}+\varepsilon$} & $\beta_{0}$ & $0,3547^{\mathrm{ns}}$ & $0,00015^{\mathrm{ns}}$ & $0,1909 *$ \\
\hline & $\beta_{1}$ & $1,3440 * * *$ & $3,1064 * * *$ & $1,4870 * * *$ \\
\hline $\mathrm{AF}=\beta_{1} * \mathrm{~L}+\varepsilon$ & $\beta_{1}$ & $17,8090 * * *$ & $69,6469 * * *$ & $48,3108 * * *$ \\
\hline \multirow{2}{*}{$\mathrm{AF}=\beta_{0}+\beta_{1} * \mathrm{~L}+\varepsilon$} & $\beta_{0}$ & $-9,9740 * * *$ & $-22,0555 * * *$ & $-88,5042 * * *$ \\
\hline & $\beta_{1}$ & $23,6969 * * *$ & $80,4902 * * *$ & $95,4177 * * *$ \\
\hline \multirow{2}{*}{$\mathrm{AF}=\beta_{0} * \mathrm{~L}^{\beta 1}+\varepsilon$} & $\beta_{0}$ & $14,2353 * * *$ & $60,3173 * * *$ & $17,3084 * * *$ \\
\hline & $\beta_{1}$ & $1,4039 * * *$ & $1,1955^{* * *}$ & $2,4883 * * *$ \\
\hline
\end{tabular}

*,***, ns Valores significativos a 5 e $0,1 \%$ e não significativo a $5 \%$, respectivamente pelo teste $\mathrm{t}$.
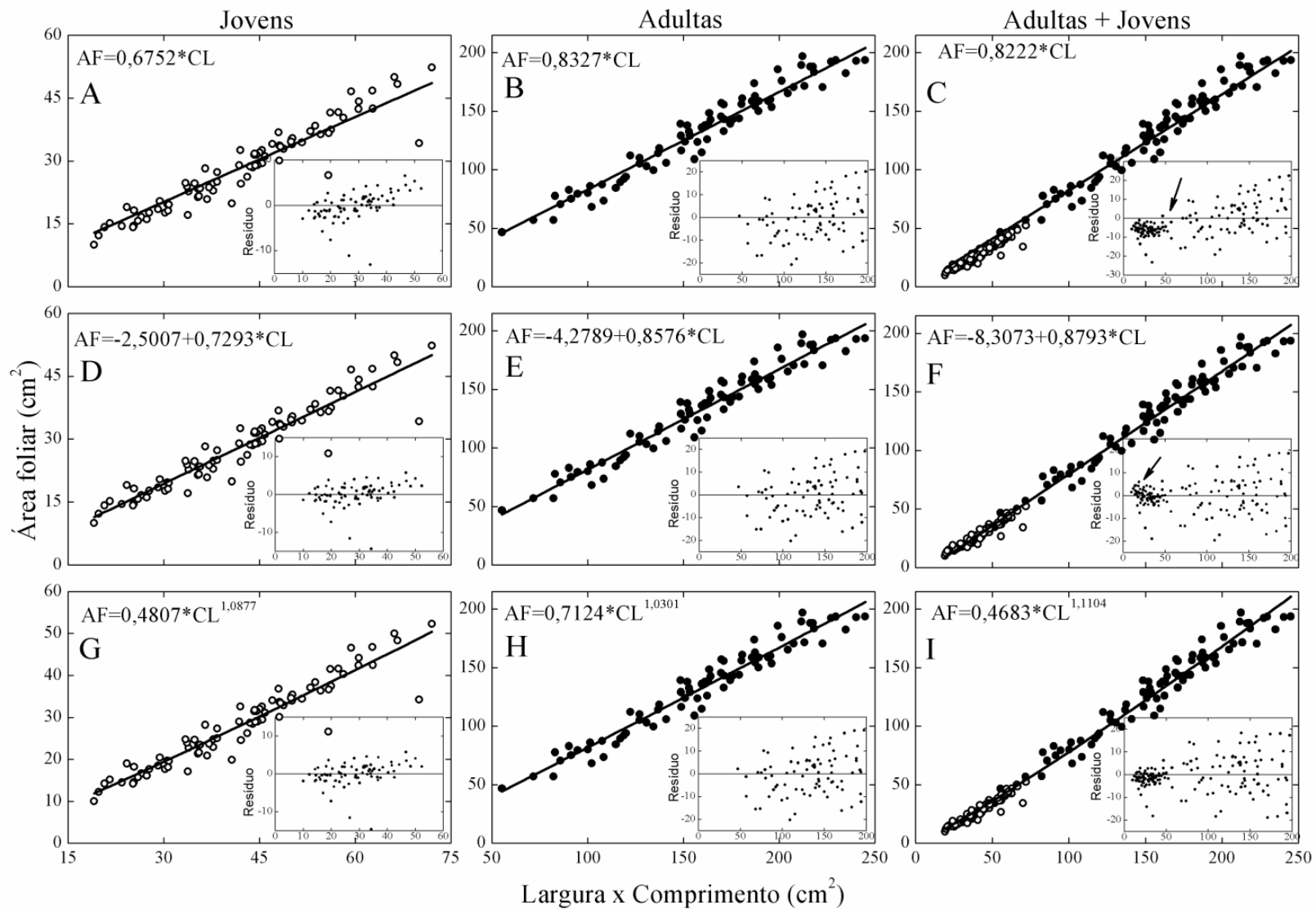

Figura 1. Relação entre a área foliar $(\mathrm{AF})$ e o produto entre o comprimento e a largura $(\mathrm{CL})$ dos folíolos para plantas jovens (A, D e $\mathrm{G})$ adultas (B, E e H) e ambas (C, F, e I) de macaúba, usando modelo polinomial de primeira ordem sem (A, B e C) e com (D, E e F) intercepto e potencial (G, H e I). O padrão de dispersão de resíduos para os respectivos modelos e idade fenológica das plantas é mostrado nas inserções. 
Tabela 2. Modelos, coeficiente de determinação $\left(\mathrm{R}^{2}\right)$, relação entre área (AF) observada e estimada (AFO/AFE) dos folíolos, soma dos quadrados do resíduo (SQr), das equações da área foliar dos folíolos de macaúba, em função do comprimento (C), largura (L) e produto de ambos (CL), em plantas jovens (J) adultas (A) e ambas (JA).

\begin{tabular}{lccccccccc}
\hline \multirow{2}{*}{ Modelo } & \multicolumn{3}{c}{$\mathbf{R}^{2}$} & \multicolumn{3}{c}{ AFO/AFE } & \multicolumn{3}{c}{ SQr } \\
\cline { 2 - 9 } & $\mathrm{J}$ & $\mathrm{A}$ & $\mathrm{JA}$ & $\mathrm{J}$ & $\mathrm{A}$ & $\mathrm{JA}$ & $\mathrm{J}$ & $\mathrm{A}$ & $\mathrm{JA}$ \\
\hline $\mathrm{AF}=\beta_{1}{ }^{*} \mathrm{CL}+\varepsilon$ & 0,90 & 0,95 & 0,98 & 0,98 & 0,99 & 0,91 & 767 & 6.359 & 10.753 \\
$\mathrm{AF}=\beta_{0}+\beta_{1}{ }^{*} \mathrm{CL}+\varepsilon$ & 0,90 & 0,95 & 0,98 & 1,00 & 1,00 & 1,01 & 726 & 6.260 & 7.393 \\
$\mathrm{AF}=\beta_{0}{ }^{*} \mathrm{CL}{ }^{\beta 1}+\varepsilon$ & 0,90 & 0,95 & 0,98 & 1,00 & 1,00 & 0,98 & 729 & 6.286 & 7.832 \\
$\mathrm{AF}=\beta_{1}{ }^{*} \mathrm{C}+\varepsilon$ & 0,37 & 0,25 & 0,83 & 0,98 & 0,98 & 0,85 & 4.801 & 86.830 & 105.494 \\
$\mathrm{AF}=\beta_{0}+\beta_{1}{ }^{*} \mathrm{C}+\varepsilon$ & 0,39 & 0,46 & 0,83 & 1,00 & 1,00 & 1,04 & 4.651 & 62.786 & 88.202 \\
$\mathrm{AF}=\beta_{0}{ }^{*} \mathrm{C}^{\beta 1}+\varepsilon$ & 0,39 & 0,46 & 0,85 & 1,00 & 0,98 & 1,07 & 4.634 & 62.636 & 82.759 \\
$\mathrm{AF}=\beta_{1}{ }^{*} \mathrm{~L}+\varepsilon$ & 0,66 & 0,88 & 0,35 & 0,97 & 0,98 & 0,89 & 2.532 & 12.998 & 362.655 \\
$\mathrm{AF}=\beta_{0}+\beta_{1}{ }^{*} \mathrm{~L}+\varepsilon$ & 0,71 & 0,89 & 0,48 & 1,01 & 1,00 & 0,91 & 2.185 & 10.984 & 293.000 \\
$\mathrm{AF}=\beta_{0}{ }^{*} \mathrm{~L}^{\beta 1}+\varepsilon$ & 0,71 & 0,89 & 0,52 & 1,00 & 1,00 & 1,10 & 2.141 & 10.805 & 265.843 \\
\hline
\end{tabular}

A soma dos quadrados do resíduo (SQr) foi menor no modelo " $\mathrm{AF}=\beta_{0}+\beta_{1}{ }^{*} \mathrm{CL}$ ", seguido pelo " $\mathrm{AF}=$ $\beta_{0}{ }^{*} \mathrm{CL}^{\beta 1}$ " e, por último, o "AF= $\beta_{1}{ }^{*} \mathrm{CL}$ ", em ambas as idades fenológicas das plantas amostradas (Tabela 2). Todavia, os dois primeiros modelos apresentaram $\mathrm{SQr}$ muito próximos e $\mathrm{R}^{2}$ idênticos. Embora o modelo "AF $=\beta_{1}{ }^{*} \mathrm{CL}$ " apresente $\mathrm{R}^{2}$ idêntico aos demais, esse possui $\mathrm{SQr}$ maior em relação aos demais, o que sugere menor precisão do modelo, ou seja, não é indicado para estimar área dos folíolos em Acrocomia aculeata.

Em relação à idade fenológica das plantas, os modelos " $\mathrm{AF}=\beta_{0}+\beta_{1}{ }^{*} \mathrm{CL}$ " e " $\mathrm{AF}=\beta_{0}{ }^{*} \mathrm{CL} \mathrm{L}^{\beta 1}$ " foram mais precisos, pois apresentaram valores de $\mathrm{AFO} /$ $\mathrm{AFE}=1,00$, para plantas jovens e adultas separadamente ou próximo a esse quando usadas ambas as idades em uma mesma equação. Porém, ao analisar o valor de $\mathrm{R}^{2}$, a união (jovens e adultas) mostrou maior coeficiente de determinação, seguida pelas plantas adultas e por último as jovens. Todavia, é importante utilizar o modelo conforme a idade fenológica da planta, uma vez que as dimensões dos folíolos provenientes de plantas jovens e adultas, praticamente, não se sobrepõem. Os modelos com ambas as idades apresentaram melhor coeficiente de determinação $(0,98)$, entretanto, a escolha do modelo não deve basear-se em um único parâmetro, no caso, elevado $\mathrm{R}^{2}$.

Ao analisar a distribuição dos resíduos nas inserções da Figura 1, observa-se que o modelo "AF $=\beta_{1}{ }^{*} \mathrm{CL}$ " (Figura 1C), quando utilizado para ambas as idades fenológicas unidas, superestimou as áreas dos folíolos de menor área, inferior a $50 \mathrm{~cm}^{2}$, uma vez que os resíduos se concentraram abaixo de zero. Esses resultados demostram que o uso de folíolos de diferentes idades fenológicas não é indicado para esse modelo. Aliado a isso, a relação $\mathrm{AFO} / \mathrm{AFE}=0,91$ também corrobora com a superestimação, proporcionada pelo modelo aos folíolos provenientes de plantas jovens. Similar resultado é observado no modelo " $\mathrm{AF}=\beta_{0}+\beta_{1}{ }^{*} \mathrm{CL}$ " (Figura 1F), todavia, nesse caso, é menos expressivo, pois subestima e abrange folíolos com área inferior a cerca de $20 \mathrm{~cm}^{2}$. Antunes et al. (2008) ao testar modelos para estimar área foliar em café observou que o modelo "AF $=\beta_{0}+\beta_{1}{ }^{*} \mathrm{CL}$ ", causou subestimativa da área foliar nos menores valores de área foliar, corroborando com o presente estudo. Segundo os autores, o modelo " $\mathrm{AF}=\beta_{0}{ }^{*} \mathrm{CL}^{\beta 1}$ " apresentou boa dispersão do resíduo, resultado semelhante a esse estudo.

Com relação ao tamanho e representatividade da amostra, observa-se que o erro padrão dos $\beta_{0}$ e $\beta_{1}$, independente do modelo utilizado, bem como da idade fenológica da planta, diminuiu conforme o aumento no tamanho da amostra, sendo estabilizado após cerca de $75 \%$ do tamanho final da amostragem, ou seja, 60 folíolos quando as idades analisadas em separado e 120 quando analisadas em conjunto (Figuras 2 e 3). Antunes et al. (2008) avaliaram o tamanho adequado da amostra para ajustar um modelo para estimar a área foliar em café e obtiveram como resultado um número mínimo de 200 folhas, o que representa cerca de $13 \%$ do tamanho total da amostra que foi de 163 folhas. Desta forma, pode-se afirmar que o número de amostras utilizadas no presente estudo foi adequado. 
Observa-se, assim, que o tamanho da amostra foi adequado para um ajuste preciso de um modelo estatístico visando estimar a área foliolar em macaúba, sejam elas jovens ou adultas. Dentre os modelos testados, o potencial foi o melhor, bem como o uso da combinação de C e L.
Assim, demonstra-se ser possível acompanhar a evolução da área foliar independente da idade fenológica das plantas de macaúba, além de não causar danos à área foliar, visto que a espécie tem baixa taxa de crescimento em área foliar, especialmente nas fases iniciais de crescimento.
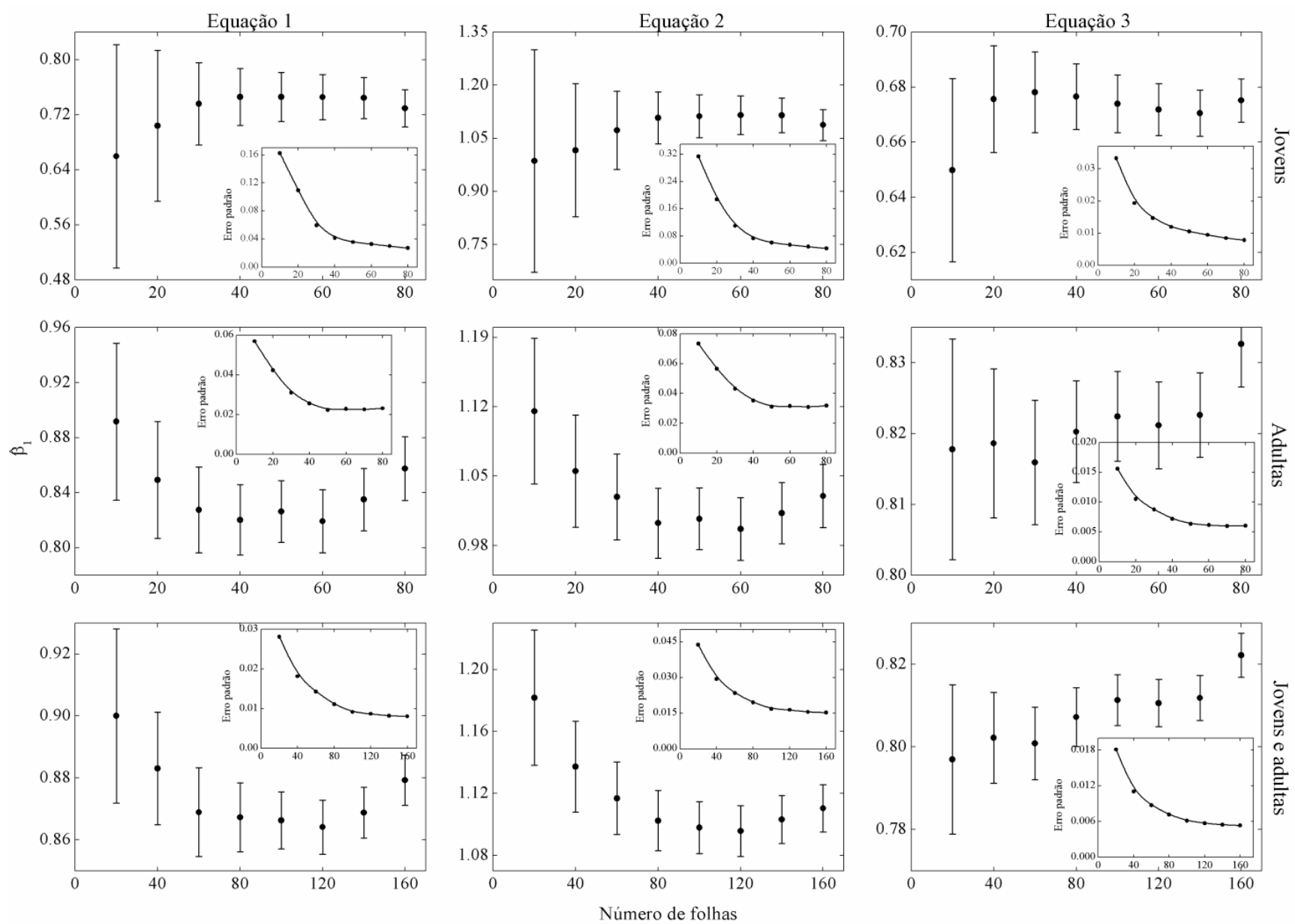

Figura 2. $\beta_{1}$ estimado para os modelos polinomial de primeira ordem com (Equação 1) e sem (Equação 2) intercepto e potencial (Equação 3), em função do tamanho da amostra. As barras verticais representam o erro padrão das médias. Na inserção, o erro padrão em função do número de amostras (folíolos). 

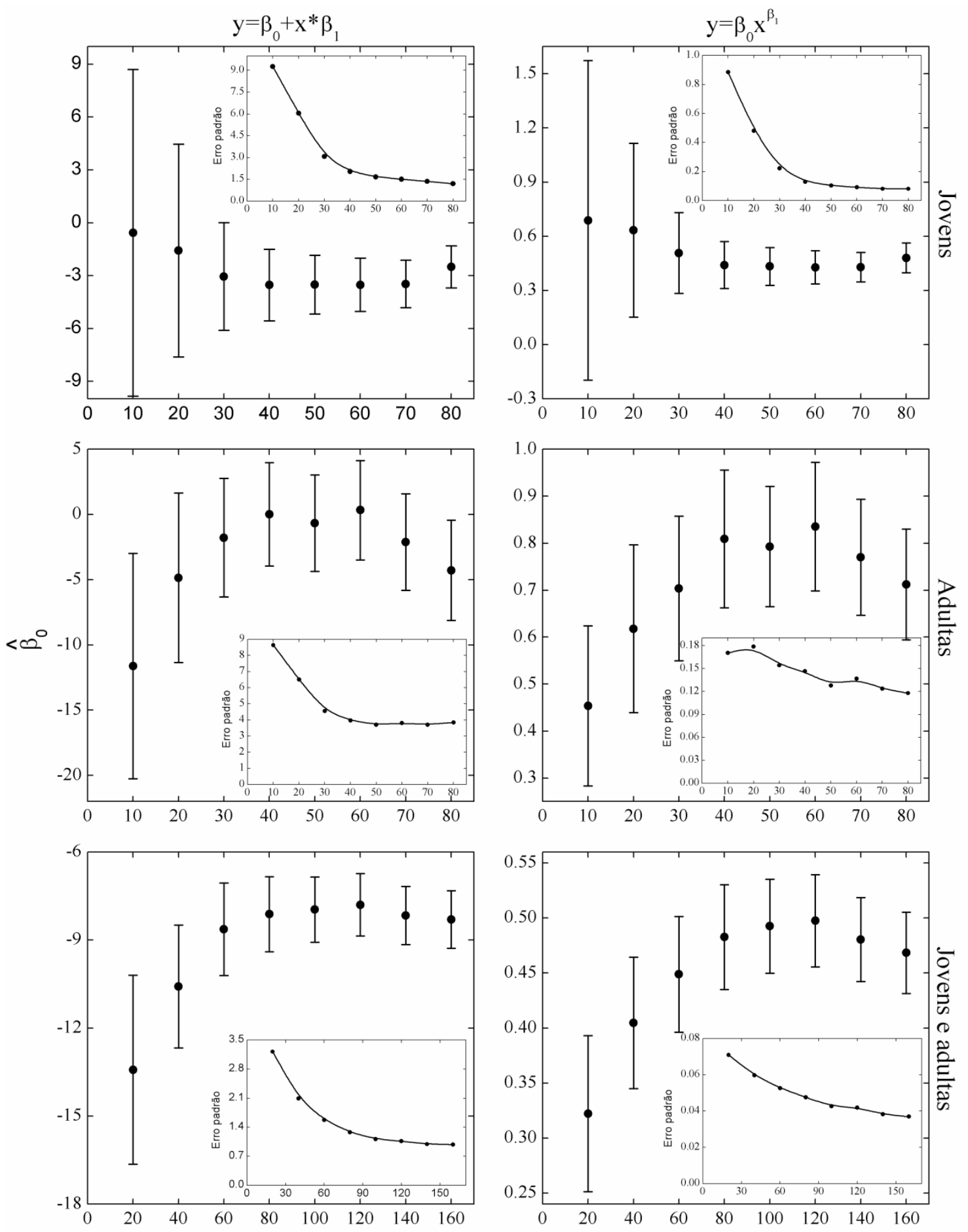

Número de folhas

Figura 3. $\beta_{0}$ estimado para os modelos polinomial de primeira ordem com intercepto e potencial, em função do tamanho da amostra. As barras verticais representam o erro padrão das médias. Na inserção o erro padrão em função do número de amostras (folíolos). 


\section{Conclusões}

É possível obter estimativas precisas de área foliar de folíolos de Acrocomia aculeata empregando modelos de regressão.

$\mathrm{O}$ produto entre o comprimento e a largura ao meio do folíolo tem correlação significativa com a área foliar dos folíolos de Acrocomia aculeata.

A equação $\mathrm{AF}=0,4683^{*} \mathrm{CL}^{1,1104}$ pode ser utilizada para estimar a área foliar de folíolos de Acrocomia aculeata, independente da idade fenológica das plantas.

Os resultados do estudo demostram ainda ser possível a estimativa da área foliolar de forma segura, sem a necessidade de destruição das folhas ou das plantas de Acrocomia aculeata, sendo isso de extrema utilidade em experimentos que acompanham a evolução de área foliar.

\section{Referências}

ANTUNES, W. C.; POMPELli, M. F.; CARRETERO, D. M.; DaMATTA, F. M. Allometric models for non-destructive leaf area estimation in coffee (Coffea Arabica and Coffea canephora). Annals of Applied Biology, Warwick, v. 153, n. 1, p. 33-40, mar. 2008. DOI: http://dx.doi.org/10.1111/j.1744-7348.2008.00235.x

BIANCO, S.; PITELLI, R. A.; BIANCO, M. S. Estimativa da área foliar de Brachiaria plantaginea usando dimensões lineares do limbo foliar. Planta Daninha, Campinas, v. 23, n. 4, p. 597-601, out./dez. 2005. DOI: http://dx.doi.org/10.1590/S0100-83582005000400006

BIANCO, S.; PITELLI, R. A.; CARVALHO, L. B. Estimativa da área foliar de Cissampelos glaberrima usando dimensões lineares do limbo foliar. Planta Daninha, Viçosa, MG, v. 20, n. 3, p. 353-356, dez. 2002. http://dx.doi.org/10.1590/S0100-83582002000300004

BOSCO, L. C.; BERGAMASCHI, H.; CARDOSO, L. S.; PAULA, V. A.; CASAMALI, B. Seleção de modelos de regressão para estimar a área foliar de macieiras 'Royal Gala' e 'Fuji Suprema' sob tela anti-granizo e em céu aberto. Revista Brasileira de Fruticultura, Jaboticabal, v. 34, n. 2, p. 504-14, Jun. 2012. DOI: http://dx.doi. org/10.1590/S0100-29452012000200024

CAMPOSTRINI, E.; YAMANISHI, O. K. Estimation of papaya leaf area using the central vein length. Scientia Agricola, Piracicaba, v. 58, n. 1, p. 39-42, Jan./Mar. 2001. DOI: http://dx.doi.org/10.1590/ S0103-90162001000100007
GONÇALVES, C. A. A.; CHALFUN, N. N. J.; REGINA, M. A.; ALVARENGA, A. A.; SOUZA, M. T.; ABRAHÃO, E. Estimativa de área foliar da videira (Vitis Labrusca L. cv. Folha de figo) sobre diferentes porta-enxertos. Ciência e Agrotecnologia, Lavras, v. 26, n. 3, p. 500-4, maio/jun. 2002.

LIMA, C. J. G. S.; OLIVEIRA, F. A.; MEDEIROS, J. F.; OLIVEIRA, M. K. T.; OLIVEIRA FILHO, A. F. Modelos matemáticos para estimativa da área foliar de feijão caupi. Caatinga, Mossoró, v. 21, n. 1, p. 120-127, jan./mar. 2008.

LOPES, C. M.; ANDRADE, I.; PEDROSO, V.; MARTINS, S. Modelos empíricos para estimativa da área foliar da videira na casta Jaen. Ciência e Técnica Vitivinícola, Dois Portos, v. 19, n. 2, p. 61-75, 2004.

MALAGI, G.; CITADIN, I.; SCARIOT, S.; REIS, L. Método não destrutivo para determinação da área foliar da videira, cultivar BRS-Violeta. Revista Brasileira de Fruticultura, Jaboticabal, v. 32, n. 4, p. 1250-1254, dez. 2010. DOI: http://dx.doi.org/10.1590/ S0100-29452011005000005

MOTA, C. S.; CORRÊA, T. R.; GROSSI, J. A. S.; CASTRICINI, A.; RIBEIRO, A. S. Exploração sustentável da macaúba para produção de biodiesel: colheita, pós-colheita e qualidade dos frutos. Informe Agropecuário, Belo Horizonte, v. 32, n. 265, p. 41-51, nov./dez. 2011.

NAKAMURA, S.; NITTA, Y.; WATANABE, M.; GOYO, Y. Analysis of leaflet Shape and area for improvement of leaf area estimation method for sago palm (Metroxylon sagu Rottb.). Plant Production Science, v. 8, n. 1, p. 24-31, abr. 2005.

NASCIMENTO, I. B.; FARIAS, C. H. A.; SILVA, M. C. C.; MEDEIROS, J. F.; SOBRINHO, J. E.; NEGRIROS, M. Z. Estimativa da área foliar do meloeiro. Horticultura Brasileira, Brasília, DF, v. 20, n. 4, p. 555-558, dez. 2002. DOI: http://dx.doi. org/10.1590/S0102-05362002000400009

SEPÚLVEDA, R. G.; KLIEWER, W. M. Estimation of leaf area of two grapevine cultivars (Vitis vinifera L) using laminae linear measurements and fresh weight. American Journal of Enology and Viticulture, Davis, v. 34, n. 4, p. 221-226, Dez. 1983.

STATSOFT INC. Statistica data analysis system version 10. Tulsa: Statsoft Inc., 2012.

WELLES, J. M. Some indirect methods of estimating canopy structure. In: GOEL, N. S.; NORMAN, J. M. Instrumentation for studying vegetation canopies for remote sensing in optical and thermal infrared regions. London: Harwood Academ Publishers, 1990. v. 5. p. 31-43. 